\title{
Acknowledgements from the Editorial Office
}

Physics is one of the most important and fundamental scientific disciplines. It aims to understand the behaviours of matter. The development of physics in 17-19 centuries has established the foundation of modern science.

Today, Physics is still one of the most attractive sciences for researchers in the area of electromagnetism, solid state physics, nuclear physics, and space physics. The development of experiment and theory in physics has led to the applications in appliances like TV and smart phone, in flying aircraft as well as in energy, environment and health, which is so called applied physics. Applied physics has become one of the important disciplines in physics due to its importance for applications.

There are many physics journals published. However, there are few for open access. Hence, we are thinking to launch the new journal "Journal of Advances in Applied Physics", which will be dedicated to the latest advancements in applied physics, and provide an online publishing platform for physics to share and exchange their research results and opinions worldwide.

Like all the other journals of the Isaac Scientific Publishing, this journal will follow the open access policy and will be peer reviewed.

It is a long journey to prepare the publication of the journal. We carefully invited renowned editorial board members and the editorial board was formed by the end of 2017. With another whole year of preparation, the first issue of the journal is finally released.

For the success of this publishing, great appreciation is expressed to our Editor-in-Chief, Prof. Jiabao Yi from the University of Newcastle, and to all the editorial board members! Your great effort guarantees the right direction of the journal and the quality of every paper. We also appreciate the efforts from all the peer reviewers. We rely upon the commitment, expertise and judgement of our reviewers to maintain the high standard of our papers. Our success is impossible without the help of the reviewers.

We are also grateful to the authors who trusted us and submitted their manuscripts to this newly created journal. We will continue to work hard and smart, and cooperate with you to create a high standard, successful journal.

Journal of Advances in Applied Physics (JAAP) Editorial Office

Isaac Scientific Publishing 